\title{
Origin of the multiferroic-like properties of $\mathrm{Er}_{2} \mathrm{CoMnO}_{6}$
}

\author{
J. Blasco ${ }^{1, a^{*}}$, G. Subías ${ }^{1, b}$, J. García $a^{1, c}$, J. Stankiewicz ${ }^{1, d}$, J. A. Rodríguez- \\ Velamazán $^{1,2, e}$, C. Ritter ${ }^{2, f}$ and J. L. García-Muñoz ${ }^{3, g}$ \\ ${ }^{1}$ Instituto de Ciencia de Materiales de Aragón, Departamento de Física de la Materia \\ Condensada, CSIC-Universidad de Zaragoza, E-50009 Zaragoza, Spain \\ ${ }^{2}$ ILL-Institut Laue Langevin, 71, Avenue des Martyrs, 38042 Grenoble Cedex, France. \\ ${ }^{3}$ Institut de Ciència de Materials de Barcelona, CSIC, Campus univ. de Bellaterra, E-08193 \\ Bellaterra, Spain \\ ajbc@unizar.es, ’gloria@unizar.es, cjgr@unizar.es, djolanta@unizar.es, ’velamazan@ill.es, \\ ritter@ill.eu, ${ }^{9}$ garciamunoz@icmab.es \\ * corresponding author
}

Keywords: Double perovskite, multiferroic, metamagnetic transition, depolarization current, ferromagnetism, neutron diffraction.

\begin{abstract}
We report on the magnetoelectric properties of $\mathrm{Er}_{2} \mathrm{CoMnO}_{6}$. This compound adopts the structure of a double perovskite with a strong monoclinic distortion. Our specimen exhibits a nearly perfect Co-Mn order. It undergoes a ferromagnetic transition at $\mathrm{T}_{\mathrm{C}} \sim 70 \mathrm{~K}$ due to the $\mathrm{Co}^{2+}-\mathrm{O}-\mathrm{Mn}^{4+}$ ferromagnetic superexchange interaction. Below $30 \mathrm{~K}$, the $\mathrm{Er}^{3+}$ moments start to order antiferromagnetically to the $\mathrm{Co} / \mathrm{Mn}$ sublattice. Pyroelectric measurements reveal electrical polarization at low temperature but its strong dependence on the heating rate indicates the lack of a spontaneous ferroelectricity. Instead, electric polarization is derived from thermally stimulated depolarization currents.
\end{abstract}

\section{Introduction}

There is a recent interest in the study of multiferroic properties in double perovskites. First principles density-functional theory calculations predicted an E-type magnetic ground state for double perovskites with heavy rare earth atoms [1,2]. It is characterized by $\uparrow \uparrow \downarrow \downarrow$ spin chains in a particular direction. Such a magnetic order breaks the inversion symmetry and allows a ferroelectric polarization perpendicular to the chain direction. This mechanism was proposed to explain the emergence of electric polarization in both $\mathrm{Y}_{2} \mathrm{CoMnO}_{6}$ [2,3] and $\mathrm{Sm}_{2} \mathrm{CoMnO}_{6}$ [4]. However, E-type order has only been determined in $\mathrm{Lu}_{2} \mathrm{CoMnO}_{6}$ and $\mathrm{Yb}_{2} \mathrm{CoMnO}_{6}$ so far [5,6]. Therefore, the study of $\mathrm{R}_{2} \mathrm{CoMnO}_{6}$ ( $\mathrm{R}=$ lanthanide) compounds is of particular interest in order to determine precisely the magnetic ground state of these compounds and the occurrence of multiferroic properties.

In this paper, we report our studies on magnetoelectric properties of $\mathrm{Er}_{2} \mathrm{CoMnO}_{6}$ (ECMO). Macroscopic measurements reveal the existence at low temperature of both, spontaneous magnetization and electric polarization (P), which are promising properties for its use as multiferroic material. However, our study demonstrates that while magnetism comes from the ferromagnetic order of Co and Mn moments, $\mathrm{P}$ is an extrinsic effect arising from reorientation of defect dipoles.

\section{Experimental section}

ECMO was prepared using ceramic procedures. Stoichiometric amounts of $\mathrm{Er}_{2} \mathrm{O}_{3}, \mathrm{Co}_{3} \mathrm{O}_{4}$ and $\mathrm{Mn}_{2} \mathrm{O}_{3}$ with nominal purities higher than $99.9 \%$ were mixed, ground and heated overnight at $900^{\circ}$ 
C. They were then ground again, pressed into pellets and sintered at $1050^{\circ} \mathrm{C}$ in air for $1 \mathrm{~d}$. The pellets were reground, repressed and sintered at $1250^{\circ} \mathrm{C}$ for $2 \mathrm{~d}$, followed by a slow cooling at $0.1^{\circ} \mathrm{C} / \mathrm{min}$ to improve the cationic ordering at the perovskite B-site [7]. X-ray diffraction patterns at room temperature agree with a single-phase perovskite.

Neutron diffraction experiments were carried out at the high-flux reactor of the ILL using the high intensity diffractometer D1B $(\lambda=2.52 \AA)$ and the high resolution powder diffractometer D2B $(\lambda=1.594 \AA)$. Structural refinements were made using the Fullprof program [8].

Magnetic measurements were carried out between 2 and $300 \mathrm{~K}$ using commercial Quantum Design (SQUID and PPMS) magnetometers. The dielectric measurements were carried out in a He cryostat in the temperature range from 5 to $300 \mathrm{~K}$, using a sintered disc with diameter of $7.9 \mathrm{~mm}$ and thickness of $0.85 \mathrm{~mm}$. Silver paint was applied for proper electrical contact. The complex dielectric permittivity of the samples was measured using an impedance analyzer (Wayne Kerr Electronics 6500B) in the frequency range between $10 \mathrm{~Hz}$ and $5 \mathrm{MHz}$. Pyroelectric current was measured with a Keithley 2635B electrometer by warming the sample at a constant rate with values ranging between 1 and $10 \mathrm{~K} / \mathrm{min}$. The change in the electrical polarization was obtained by integrating the pyroelectric current as a function of time. The electric field was applied at $150 \mathrm{~K}$ followed by cooling at a rate of $10 \mathrm{~K} / \mathrm{m}$ down to $5 \mathrm{~K}$. Then, the field was removed and the stabilization of the polarization was reached after shorting the circuit for $15 \mathrm{~m}$.

\section{Results}

The x-ray diffraction patterns of ECMO can be indexed in the $P 2_{1} / n$ space group which is one of the most common distorted structures of the double perovskites exhibiting cationic ordering at the perovskite B-site. Accurate Rietveld refinements can be obtained using this structural model but the exact $\mathrm{Co} / \mathrm{Mn}$ distribution is hardly disclosed by x-ray diffraction as both cations have similar scattering factors. Thus, neutron diffraction was used for this purpose. Fig. 1 shows the refinement of the neutron pattern collected at $295 \mathrm{~K}$ (D2B). The refinement of Co/Mn occupancies in the Bsublattice confirms a high ordering degree of $\sim 94 \%$. Then, anti-site defects concentration is $\sim 6 \%$ (50\% means random $\mathrm{Co} / \mathrm{Mn}$ distribution). The refined interatomic Co-O ( 2.053 $\AA$ ) and Mn-O $(\sim 1.912 \AA)$ distances are close to the ones expected for $\mathrm{Co}^{2+}-\mathrm{O}$ and $\mathrm{Mn}^{4+}-\mathrm{O}$, respectively.
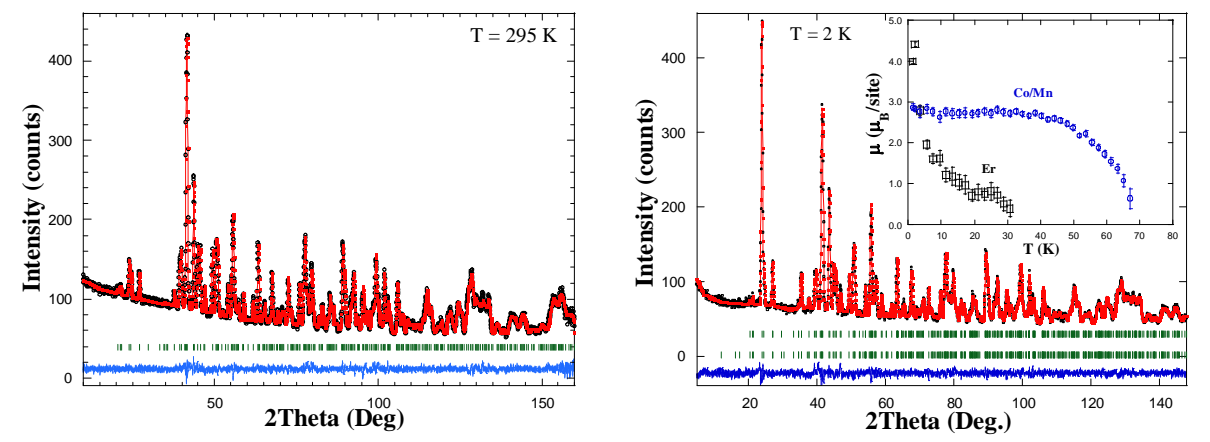

Fig 1. Rietveld refinement of the neutron powder pattern of ECMO sample at (left) $295 \mathrm{~K}$ and (right) $2 \mathrm{~K}$. Inset: The temperature dependence of refined magnetic moment for $\mathrm{Co} / \mathrm{Mn}$ and $\mathrm{Er}$ sublattices.

Significant changes are observed in the neutron patterns on cooling. Below $\mathrm{T}_{\mathrm{C}} \sim 70 \mathrm{~K}$, the growth of several diffraction peaks points at the onset of long range magnetic order. The refinements agree with a ferromagnetic (FM) coupling between $\mathrm{Mn}$ and Co moments located in the ac-plane. The refinements become very robust when the same magnetic moment is assumed for both atoms. This is expected when both cations, $\mathrm{Co}^{2+}$ and $\mathrm{Mn}^{4+}$, are in high spin state with $\mathrm{S}=3 / 2$. Below $30 \mathrm{~K}$, the intensity of some diffraction peaks undergoes sudden changes pointing to the $\mathrm{Er}^{3+}$ ordering. Fig. 1 also displays the refinement of the pattern at $2 \mathrm{~K}$ that agrees with an antiferromagnetic (AFM) coupling between Er and Co/Mn sublattices with the moments lying within the ac-plane. The refined moments at $2 \mathrm{~K}$ are 3.0 and 4.25 for $\mathrm{Co} / \mathrm{Mn}$ and $\mathrm{Er}$, respectively. The former value agrees with the theoretical one whereas the latter is well below the free ion $\mathrm{Er}^{3+}$ moment of $9 \mu_{\mathrm{B}}$, indicating an incomplete order at $2 \mathrm{~K}$. The inset of Fig 1 shows the temperature dependence of 
refined moments confirming the different behavior of both sublattices: $\mathrm{Co} / \mathrm{Mn}$ moments are saturated at $2 \mathrm{~K}$ while the Er moments are still rising at this temperature.

Magnetic measurements also disclose the presence of a magnetic transition at low temperature. Fig. 2(a) displays the temperature dependence of dc magnetization under a magnetic field of 100 Oe in zero-field cooled (ZFC) and field-cooled (FC) conditions. These measurements reveal a strong magnetic irreversibility: The ZFC branch shows a broad peak centered at $~ 57 \mathrm{~K}$ while the FC branch exhibits a ferromagnetic-like transition at $\mathrm{T}_{\mathrm{C}} \sim 68 \mathrm{~K}$ (inflection point) and a subsequent decrease at temperatures below $30 \mathrm{~K}$ which matches with the onset of the magnetic ordering in the Er-sublattice. Above the transition temperature, the sample exhibits a paramagnetic behavior obeying the Curie-Weiss law in a wide temperature range. The inset of Fig. 2(a) shows the inverse of the ac magnetic susceptibility with the linear fit to it above $120 \mathrm{~K}$. The fit yields a small and positive Weiss constant indicating the presence of ferromagnetic correlations in the paramagnetic region. The effective paramagnetic moment nicely agrees with the expected one assuming $9.59 \mu_{\mathrm{B}}$ (J-value), $3.87 \mu_{\mathrm{B}}$ (spin-only) and $4.8 \mu_{\mathrm{B}}$ (experimental value) for $\mathrm{Er}^{3+}, \mathrm{Mn}^{4+}$ and $\mathrm{Co}^{2+}$, respectively.
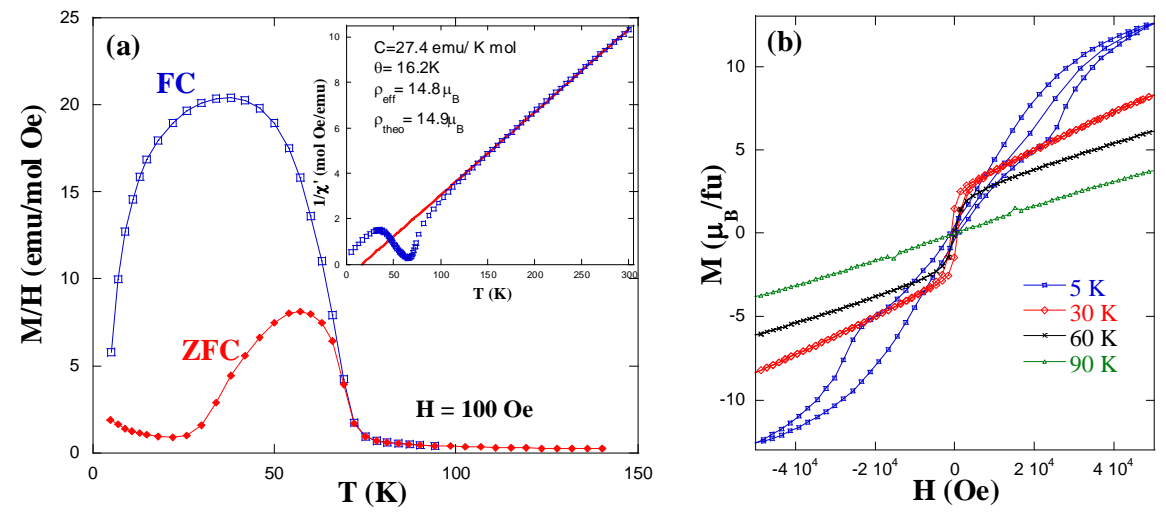

Fig 2. (a) dc magnetization vs. temperature in ZFC and FC conditions. Inset: $1 / \chi_{\mathrm{ac}}$ vs. temperature. The straight line shows the fit to a Curie-Weiss law. (b) Hysteresis loops of ECMO at the indicated temperatures.

A spontaneous magnetization below $\mathrm{T}_{\mathrm{C}}$ is clearly seen, in agreement with the $\mathrm{FM}$ coupling between $\mathrm{Co}^{2+}$ and $\mathrm{Mn}^{4+}$ moments. Hysteresis loops collected at selected temperatures are plotted in the Fig. 2(b). The curve at $90 \mathrm{~K}$ is typical of a paramagnetic system. The isotherm curve at $60 \mathrm{~K}$ shows two magnetic contributions: (i) spontaneous magnetization ascribed to the FM order in the $\mathrm{Co} / \mathrm{Mn}$ sublattice as observed by neutron diffraction, and (ii) a paramagnetic contribution ascribed to the disordered $\mathrm{Er}^{3+}$ ions that accounts for the positive slope of the loop at high fields. Similar features are observed in the loop at $30 \mathrm{~K}$, which shows no magnetic saturation. The only difference is the increase of the coercive field of the ferromagnetic phase with decreasing temperature. The hysteresis loop at $5 \mathrm{~K}$ shows a unique behavior. At low fields it adopts a wasp-waisted shape. On increasing the magnetic field (either positive or negative), a plateau-like anomaly is noticeable at $\sim 20-25$ kOe. Magnetic saturation is not reached at $50 \mathrm{kOe}$ but the moment at this field is $\sim 12.6$ $\mu_{\mathrm{B}} / \mathrm{fu}$, a value much higher than the expected one for a fully saturated Mn-Co sublattice $\left(6 \mu_{\mathrm{B}} / \mathrm{fu}\right)$. Moreover, this value overcomes the antiferromagnetic coupling between Er and Co/Mn moments (12 $\mu_{\mathrm{B}} / \mathrm{fu}$ ). Therefore, the only explanation is a ferromagnetic coupling of both sublattices induced by the external field. In this way, the plateau-like anomaly marks a metamagnetic transition with a reorientation of Er and Co/Mn moments yielding a FM coupling.

Electric properties have also been characterized in ECMO. Fig. 3(a) shows the temperature dependence of its dielectric constant $\left(\varepsilon_{\mathrm{r}}{ }_{\mathrm{r}}\right)$ and dielectric loss factor $(\tan \delta)$ for this compound. The plots illustrate the ac dielectric dispersions for different frequencies. $\varepsilon_{\mathrm{r}}{ }_{\mathrm{r}}(\mathrm{T})$ curves exhibit a step-like increase from a low-temperature value of $\sim 10$ to huge values of $\sim 10^{3}$ at $\sim 290 \mathrm{~K}$. The step shifts to higher temperatures with increasing frequency, and is accompanied by a peak in the $\tan \delta$. Above this peak, $\tan \delta$ rises, indicating a strong electronic contribution at room temperature. Similar features in related oxides were attributed to extrinsic effects such as Maxwell-Wagner type contributions of depletion layers. We have also measured the electric polarization (P) obtained from pyroelectric currents. We find that polarization below $150 \mathrm{~K}$ increases monotonously with increasing poling field. However the onset temperature of $\mathrm{P}$ strongly depends on the heating rate as 
can be seen in Fig. 3(b). This result indicates the lack of a ferroelectric order and the occurrence of thermally stimulated depolarization current (TSDC). Heating rate dependence of the peak position in the pyroelectric current follows the Bucci equation [9]: $U / k_{B}=T^{2}{ }_{\max } \exp \left(-U / k_{B} T_{\max }\right) / \beta \tau^{0}$; where $\mathrm{U}, \mathrm{k}_{\mathrm{B}}, \beta, \tau^{0}$ and $\mathrm{T}_{\max }$ stand for activation energy, Boltzmann constant, heating rate, relaxation time and position of the TSDC peak maximum, respectively. The observed linear dependence is ascribed to relaxation of defect dipoles.
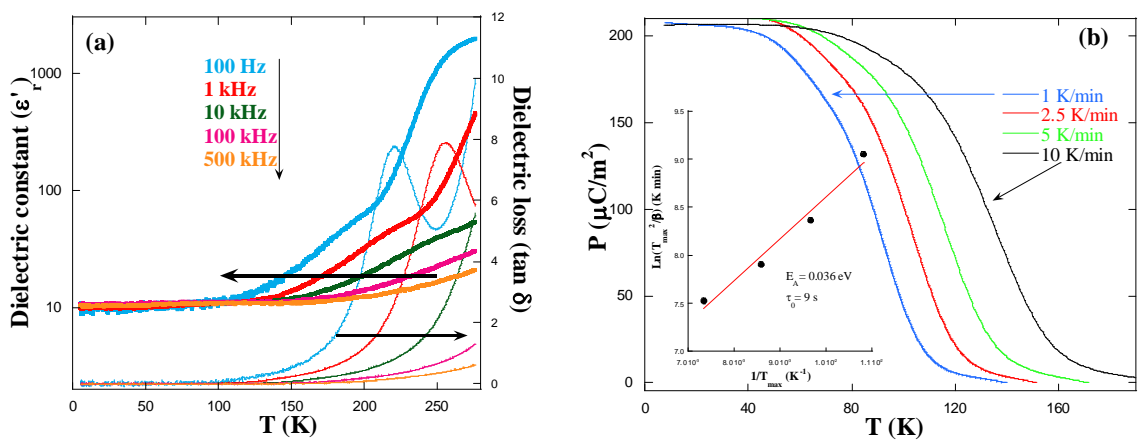

Fig 3. (a) Dielectric constant $\left(\varepsilon_{r}^{\prime}\right)$ and dielectric loss $(\tan \delta)$ vs. T for ECMO. (b) P vs. T for ECMO with different heating rates. Inset: Peak temperature of pyroelectric current vs. heating rate plotted as $\mathrm{T}_{\text {max }}^{2} / \beta$ vs $1 / T_{\max }$. The line is a fit to the Bucci equation [9].

\section{Conclusions}

ECMO can be prepared with a high level of cationic order degree. This compound exhibits a spontaneous magnetization at $\mathrm{T}_{\mathrm{C}} \sim 70 \mathrm{~K}$ and an electric polarization below $\sim 150 \mathrm{~K}$. The spontaneous magnetization comes from the FM ordering $\mathrm{Co}^{2+}$ and $\mathrm{Mn}^{4+}$ moments. Below $30 \mathrm{~K}$, $\mathrm{Er}^{3+}$ moments start to couple antiferromagnetically to $\mathrm{Co} / \mathrm{Mn}$ sublattice. The resulting magnetic structure is collinear with the propagation vector $\mathbf{k}=0$. This result evidences that E-type order is more difficult to achieve in double perovskites than initially predicted by theory.

The dielectric permittivity of ECMO does not show any anomaly associated to the occurrence of P. Its behavior suggests the presence of depletion layers and significant electron conduction at high temperature. A thorough study reveals that $\mathrm{P}$ depends on the heating rate, which is typical behavior of TSDC. Our results also indicate that $\mathrm{P}$ arises from the reorientation of defect dipoles, a feature already found in related compounds [9]. Therefore, ECMO is neither ferroelectric nor multiferroic.

Acknowledgements. We thank financial support from the Spanish MINECO (Projects No. MAT2015-68760-C2-1 and MAT2015-68760-C2-2, cofunded by ERDF from EU) and DGA (E69). We also acknowledge ILL for beam time allocation and the use of SAI from Universidad de Zaragoza. J. A. Rodríguez-Velamazán acknowledges CSIC for JAEdoc contract.

\section{References}

[1] S. Kumar et al., Phys. Rev. B 82 (2010) 134429.

[2] C. Y. Ma et al., Phys. Chem. Chem. Phys. 17 (2015) 20961.

[3] G. Sharma et al., Appl. Phys. Lett. 103 (2013) 012903.

[4] L. Wang et al., J. Appl. Phys. 117 (2015) 17 D914.

[5] S. Yañez-Vilar et al., Phys. Rev. B 84 (2011) 134427.

[6] J. Blasco et al., Appl. Phys. Lett. 107 (2015) 012902.

[7] T. Kyômen et al., Chem. Mater. 15 (2004) 4798.

[8] J. Rodriguez-Carvajal, Physica. B 192 (1993) 55.

[9] J. Blasco et al., Phys. Rev. B 93 (2016) 214401. 